\title{
Dynamic Model of Paclitaxel Biosynthesis Suggests That the Key Enzyme Is Taxadiene 5alpha-Hydroxylase in Taxuschinensis Cell Suspension Culture
}

\author{
S.S. Zhu, Z.Q. Miao \\ College of Agriculture and Biology \\ Shanghai Jiaotong University \\ Shanghai, China
}

\begin{abstract}
Taxus suspension cell cultures are a sustainable source of paclitaxel. In this study, transcript profiles of $T$. chinensis cells were analysed systematically by RT-qPCR to investigate the transcriptional dynamics of Taxus cell during cultures. Correlation between the expression levels of genes involved in paclitaxel biosynthesis enzymes and the specific synthesis rate of paclitaxel in suspension culture of Taxuschinensis had been studied by a statistic model. The model suggested that, taxadiene 5-alpha-hydroxylase, with the highest correlation coefficient, was the rate-limiting enzyme in paclitaxel biosynthetic pathway in Taxuschinensis.
\end{abstract}

Keywords-paclitaxel biosynthesis genes; relative expression; rate-limiting step

\section{INTRODUCTION}

Paclitaxel, isolated from the bark of several Taxus species, is diterpene alkaloid with remarkable anticancer propertiese specially effective against breast cancer and non-small cell lung cancer [1].The most promising and environmentally friendly way for the sustainable production of paclitaxel is provided by plant cell cultures. Though more than 20 molecular cloning of cDNA sequences encoding these enzymes have been studied [2], the genes that control the bottleneck steps of paclitaxel synthesis are still unknown. Taxadiene synthase (TS), catalysinggeranylgeranyl pyrophosphate to generate taxane skeleton, was thought as the most promising candidate, only because it is responsible for the branch point biochemical reaction and its cyclization activity is very low [3].

This study systematically detected the expression of more than20 genes involved in paclitaxel biosynthesis in Taxuschinensis suspension cultures. A model had been constructed to study the correlation between gene transcript profiling with paclitaxel synthesis kinetics in order to show light on the committed steps of paclitaxel biosynthetic.

\section{MATERIAL AND METHODS}

\section{A. Cell Culture and Paclitaxel Measurement}

The Taxuschinensis cell line was provided by Shanghai Jiaotong University. Cell cultures were grown as Miao [4] described. Paclitaxel was measured by HPLC as Yu [5]described.

\section{B. Total Rna Extraction and Cdna Preparation}

Cell samples were frozen with liquid nitrogen and stored at $-80^{\circ} \mathrm{C}$. Total RNA was extracted with Tiangen RNA extraction kit. The first-strand cDNA synthesis was carried with Prime Script ${ }^{\circledR}$ RT-PCR Real Time kit provided by Takara.

Primers were designed using primer premier 5. Tiangen Super Real PreMix Plus (SYBR Green) kit was used for RT-qPCR. The $\beta$-actin gene of Taxus cuspidate was used as a housekeeping control. The experiments were repeated three times. Data was analyzed using $2-\Delta \Delta \mathrm{CT}$ method.

\section{RESULTS AND DISCUSSION}

\section{A. Transcript Profiling}

1) The upstream pathway genes: The upstream pathway is composed of two alternative compartmental pathways: MVA pathway in cytosol and MEP/DXP pathway in plastid.
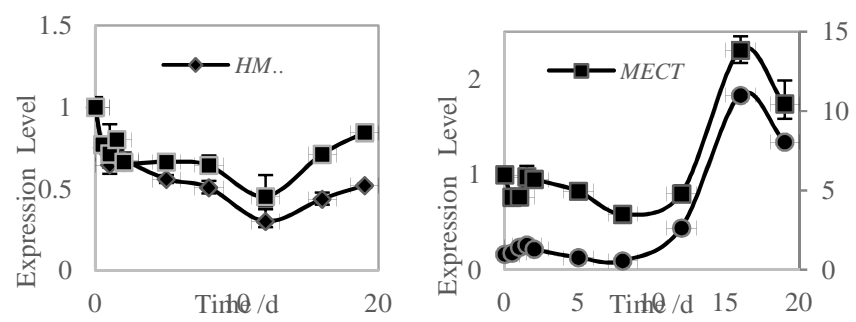

(a) HMGR and HMGS expression patterns, (b) MECT and DXS expression patterns
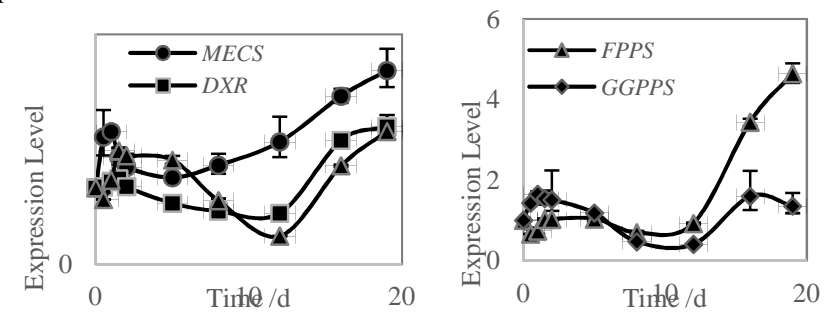

(c) MECS, DXR and HMDR expression patterns, (d) FPPS and GGPPS expression patterns

FIGURE I. GENE EXPRESSION PATTERNS OF THE UPSTREAM BIOSYNTHETIC PATHWAY OF PACLITAXEL. 
The expression of all upstream genes rapidly and slightly increased during the first 1.5 days due to cellular microenvironment disturbance in the early vaccination. And then the expression decreased slightly and the lowest decrease in transcriptional level was observed on day 8 or day 12 . The gene expression went up sharply after day 12 when nutrients have been depleted. (Figure 1)

The expression of genes involved in MVA pathway, such as HMGR and HMGS genes, were always less than the control (Figure 1a), while the expression of five genes in DXP pathway increased sharply after day 8 or 12 and up to 2-5 times more than the control. It was consistent with the theory that terpene precursor was mainly from the DXP pathway. [6].

2) The downstream genes: Following the formation of the special taxane skeleton, 18 sequential biochemical reactions, being responsible for the modification to the nucleus mainly by hydroxylation and acylation, constitute the downstream pathway.
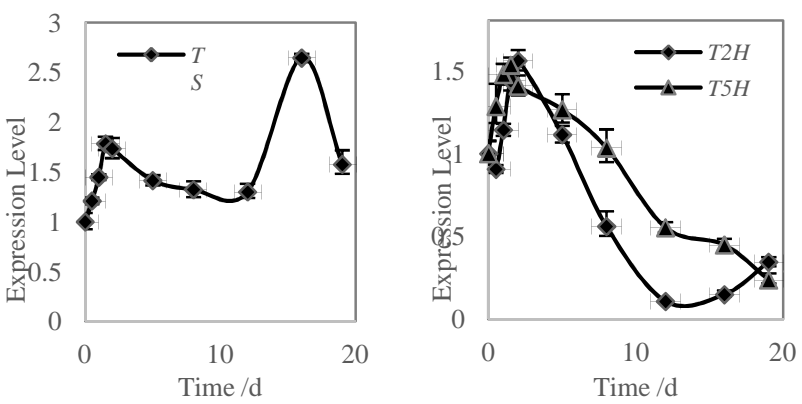

(a) TS expression patterns, (b) $\mathrm{T} 2 \mathrm{H}$ and $\mathrm{T} 5 \mathrm{H}$ expression patterns
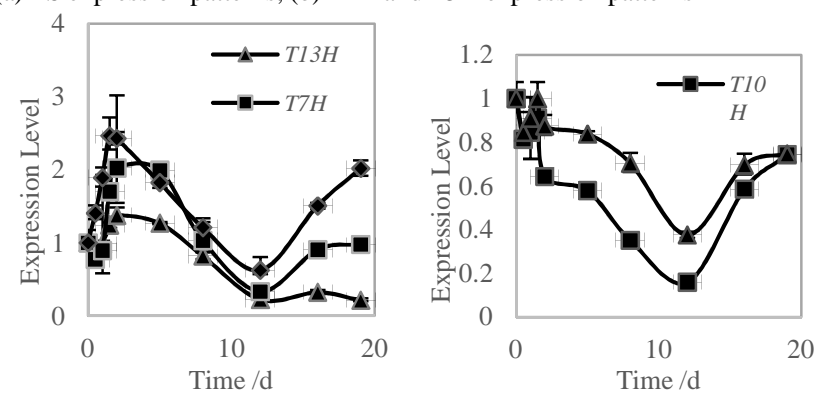

(c) $\mathrm{T} 13 \mathrm{H}, \mathrm{T} 7 \mathrm{H}$ and NADPH expression patterns, (d) $\mathrm{T} 10 \mathrm{H}$ and $\mathrm{T} 14 \mathrm{H}$ expression patterns
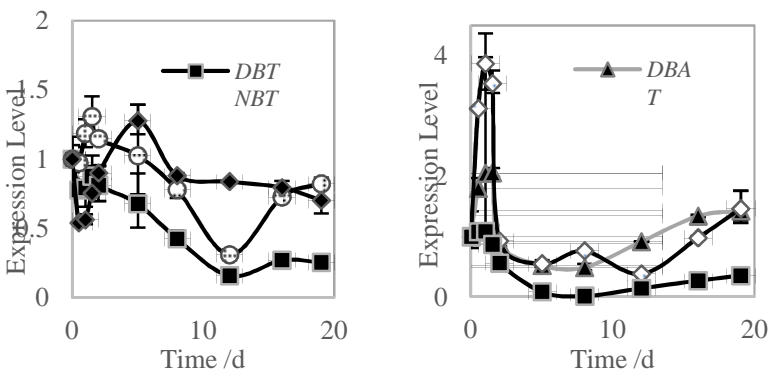

(e)DBTNBT, BAPT and TAT expression patterns (f)BAPT, PAM and TBT expression patterns

FIGURE II. EXPRESSION PATTERNS OF THE DOWNSTREAM GENES.

The highest increase in transcriptional level of TS was observed in late culture (Figure 2a).The expression patterns of cytochrome $\mathrm{P} 450$ oxygenases genes activating taxane skeleton fluctuated violently. Similarly with other genes, the expression of hydroxylase genes increased slightly in the first 2 days of culture due to cellular microenvironment disturbance (Figure 2b-d). After the lag phase, the expression of hydroxylase genes decreased rapidly after day 2 . In the late culture, their expression went up again as the second peak because paclitaxel biosynthesis rebloomed after the inhibition to the secondary metabolism reduced with the depletion of nutrients.

Comparing with the violent wave of cytochrome P450oxygenases genes, the expression of acyltransferase genes in late paclitaxel biosynthesis pathway varied little in the cell culture. The expression increased significantly in early culture but no second expression peak was observed in late culture (Figure 2e-f).

\section{B. Dynamic Model Analysis of Paclitaxel Biosynthesis}

3) Dynamic model of paclitaxelbiosynthesis: Paclitaxel specific synthesis rate was obtained:

$$
\mathrm{r}=\frac{1}{X} d p / d t
$$

where $\mathrm{x}$ stands for cell volume, $\mathrm{p}$ is for paclitaxel content, $\mathrm{t}$ is short for time.

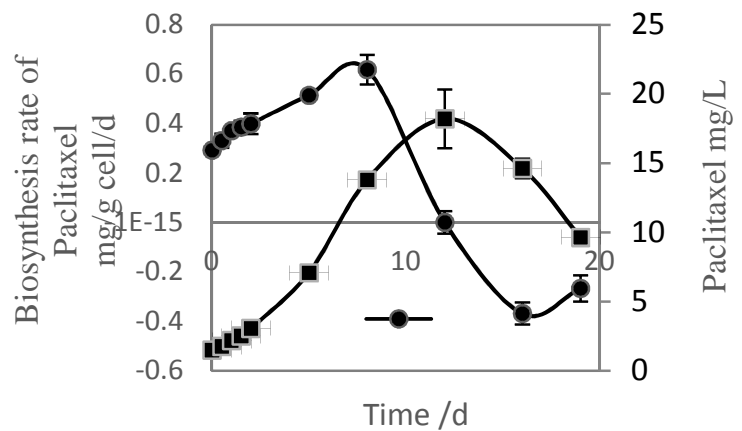

FIGURE III. PACLITAXEL CONTENT TRENDS AND PACLITAXEL SPECIFIC SYNTHESIS RATE TRENDS.

As been shown in Figure 3, the first 12 days was accumulation phase for paclitaxel synthesis and then the paclitaxel content began to decrease. The highest paclitaxel biosynthetic activity was observed around day 8 according to paclitaxel specific synthesis rate trends. Paclitaxel biosynthetic activity of cells improved gradually from vaccination to day 8 and then decreased rapidly after day 8 down to 0 in day 12 which meant paclitaxel synthesis rate and degradation rate were equal in that day. And after day 12, paclitaxel degradation rate exceeded paclitaxel synthesis.

4) The committed step model based on the correlation analysis between paclitaxel biosynthesis rate and the transcription expression of enzyme: Paclitaxel complex biosynthetic pathway can be simplified to a series of reaction dynamics system and the slowest rate-limiting steps determine the paclitaxel synthesis rate of Taxus cells. Assuming that expression level determines transcriptional level and decides the catalytic activity of enzymes, there would be positive correlation between the transcription level of the rate-limiting enzymes and paclitaxel special synthesis rate. On the other hand the correlation coefficient of non-limiting enzyme is 
little. Therefore, the committed step could be deduced based on the statistics correlation between the paclitaxel synthesis and the transcriptional of paclitaxel synthesis.

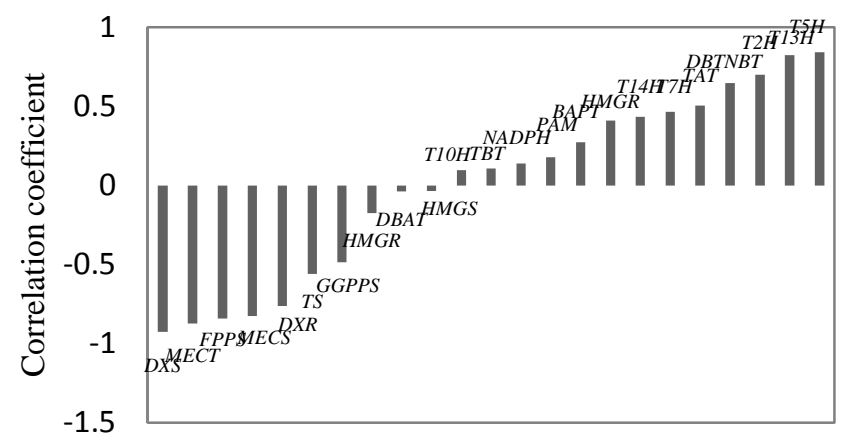

FIGURE IV.CORRELATION COEFFICIENTS OF THE RELATIVE EXPRESSION OF EACH GENE AND PACLITAXELSYNTHESIS RATE OF UNIT CELL.

As was shown in Figure 4, the correlation coefficient of TS which catalyzes the formation of taxane skeletonwas- 0.56 . The lower positive correlation was most likely because most of taxane skeleton TS catalyzed was converted into other taxane and only small part was converted to paclitaxel which was one of 300 kinds of taxane. Basing on this viewpoint, though TS is a key enzyme catalyzing the formation of taxane skeleton at topology branchpoint, it was not the rate-limiting enzyme in paclitaxel biosynthesis pathway.

PAM was predicted as a committed enzyme catalyzing the first step in the biosynthesis of the C-13 side chain of paclitaxel[7]. M.Bonfill thought that DBAT gene controlled bottle-neck step in paclitaxel production in aerial part of Taxus baccata because 10-deacetylbaccatin III poorly converted into baccatinIII by DBAT[8]. However, the correlation coefficient of PAM was as low as 0.2 and that of DBAT was -0.05 . Therefore, they were not the rate-limiting enzymes.

$\mathrm{T} 5 \mathrm{H}$ catalyzes hydroxylation at $\mathrm{C} 5$ of taxane skeleton following TS catalyzing the formation of taxane skeleton. But T5Hhad the highest correlation with paclitaxel synthesis rate $(\mathrm{r}$ $=0.84$ ). It suggested that T5H catalyzes the committed step of paclitaxel biosynthesis in Taxus Chinese cell suspension cultures.

Three enzymes with highest correlation coefficient were all cytochrome $\mathrm{P} 450$ oxygenases (T5H, T13H, T2H) and the average correlation coefficient of the six hydrogenases was 0.51 . DBTNBT, catalyzing the last acylation step of paclitaxel biosynthesis, had the most high correlation coefficient $(\mathrm{r}=0.51)$ among all the five acylases $(r=0.29)$. Hydrogenase, activating the skeleton, had higher average correlation coefficient than acylase, responsible for acetyl modification, which suggested subsequent acylation activity was high enough and taxane skeleton activating by hydroxylation was more important than the following acylation in TaxusChinens is cell suspension cultures.

The upstream pathway is composed of the biogenesis of the universal diterpenoid progenitor and the average correlation coefficient was as low as -0.50 . It was likely because there were branches for many other byproducts and most terpenoid precursors were converted non-taxaneterpene.

Comparing with the upstream, there was obviously positive correlation between transcriptional level of the downstream enzymes and the paclitaxel synthesis rate $(r=0.33)$. The possible reason was that the topological distance of these enzymes for paclitaxel was shorter and less byproducts other than paclitaxel existed so that more products are converted to paclitaxel. It suggested that strongly expressing the downstream genes, especially $\mathrm{T} 5 \mathrm{H}$, would be more beneficial for improving paclitaxel biosynthesis than strongly expressing the upstream genes.

\section{CONCLUSION}

The correlation coefficient of $\mathrm{T} 5 \mathrm{H}$, was the highest which suggest that $\mathrm{T} 5 \mathrm{H}$ was the rate-limiting enzymeof paclitaxel biosynthesis. Cytochrome P450 oxygenases, activating the skeleton, were slow and control the biosynthesis of paclitaxel. Improving the expression of hydrogenases, especially $\mathrm{T} 5 \mathrm{H}$, would give more opportunities to manipulate paclitaxel production.

\section{REFERENCES}

[1] SchiffP.B.,FantJ,HorwitzS.B.,Promotion of microtubuleassembly in vitro by paclitaxel[J]. Nature, ,277, pp. 665-667, 1979.

[2] X.L. Yu, S.S. Zhu,R.H. Chen, Z.Q. Miao, Advances on paclitaxel biosynthesis in Taxus[C]. 2013 International Conference on Biological, Medical and Chemical Engineering:HongKong,pp. 331-336, 2013.

[3] Mustafa Köksal,YinghuaJin, Robert M. Coates, Rodney Croteau,David W. Christianson. Taxadiene synthase structure and evolution ofmodular architecture in terpenebiosynthesis[J].Nature, 467, pp. 116-122, 2011.

[4] Z.Q.Miao, Z.J. Wei, J.Y. Yuan,Effects of osdmotic pressure and nutrient concentration on cell growth and Toxol production of Taxuschinesis Var Maireisuspension cultures[J].Journal of Wuxi University of Light Industry, 18(5), pp. 31-34, 1999.

[5] X.L. Yu,Z.Q. Miao, M.Y. Li, Study on the stability of Paclitaxel in water $[J]$.Joural of Shanghai JiaotongUniversity(Agricultural Science), 05, pp. 520-523, 2009.

[6] Palazon J, Cusido R.M., Bonfill M, et al Inhibition of paclitaxel and baccatin III accumulation by mevinolin and fosmidomycin in suspension cultures of Taxusbaccata [J]. Biotechnol,101(2), pp. 157-163, 2003.

[7] Paul E. Fleming, Andrew R. Knaggs, Xian-GuoHe,etal.Biosynthesis of Taxoidsmode of attachment of thePaclitaxelsidechain [J].J. Am. Chem. SOC.,116, pp. 4137-4138, 1999

[8] M. Onrubiab, E. Moyanob, M. Bonfill,etal.The relationship between TXS, DBAT, BAPT and DBTNBT gene expression and taxane production during the development of Taxusbaccataplantlets[J].Plant Science,181, pp. 282-287, 2011. 